\title{
Utilização dos serviços odontológicos por pacientes pediátricos com Síndrome de
}

\section{Down de acordo com os cuidadores}

\author{
Use of dental services by pediatric patients with Down Syndrome according to caregivers \\ Utilización de servicios dentales por pacientes pediátricos con Síndrome de Down según cuidadores
}

Recebido: 10/03/2021 | Revisado: 19/03/2021 | Aceito: 23/03/2021 | Publicado: 01/04/2021

\author{
Daniella Nunes Fragôso \\ ORCID: https://orcid.org/0000-0001-6564-2838 \\ Centro Universitário de João Pessoa, Brasil \\ E-mail: fragosodaniella@gmail.com \\ Gabriella Maria Lopes Negromonte Silva \\ ORCID: https://orcid.org/0000-0002-6095-2949 \\ Centro Universitário de João Pessoa, Brasil \\ E-mail: gabriella_negromonte17@hotmail.com \\ José Jhenikártery Maia de Oliveira \\ ORCID: https://orcid.org/0000-0003-2915-5607 \\ Centro Universitário de João Pessoa, Brasil \\ E-mail: jhenikarterymaia@gmail.com \\ Micaella Fernandes Farias \\ ORCID: https://orcid.org/0000-0002-4072-8149 \\ Centro Universitário de João Pessoa, Brasil \\ E-mail: micaellaff@gmail.com \\ Kauana da Silva Andrade \\ ORCID: https://orcid.org/0000-0002-0385-9983 \\ Centro Universitário de João Pessoa, Brasil \\ E-mail: kauanaandrade12@gmail.com \\ Nathalia Farias Dantas de Figueiredo \\ ORCID: https://orcid.org/0000-0002-8328-8446 \\ Centro Universitário de João Pessoa, Brasil \\ E-mail: nathaliafariasd@gmail.com \\ Karina Tomé Fragoso \\ ORCID: https://orcid.org/0000-0002-5647-1552 \\ Centro Universitário de João Pessoa, Brasil \\ E-mail: karinaafragooso@ hotmail.com \\ Andreia Medeiros Rodrigues Cardoso \\ ORCID: https://orcid.org/0000-0002-2682-1048 \\ Centro Universitário de João Pessoa, Brasil \\ E-mail: andreia.medeiros@unipe.edu.br
}

\begin{abstract}
Resumo
Objetivo: Avaliar a utilização dos serviços odontológicos por pacientes pediátricos com Síndrome de Down de acordo com os cuidadores. Metodologia: Realizou-se um estudo transversal na Fundação Centro Integrada de Apoio ao portador de Deficiência (FUNAD), em João Pessoa-PB. A amostra foi constituída por 49 cuidadores de pacientes com Síndrome de Down, entre 0 e 15 anos. Foram coletados dados socioeconômicos e de satisfação com o serviço ofertado por meio de um formulário elaborado de acordo com os objetivos da pesquisa. Resultados: Observou-se que $83,7 \%$ dos pacientes já foram ao dentista, com maior frequência na Unidade de Básica de Saúde (47,5\%). 97,5\% dos cuidadores demonstraram-se satisfeitos com a assistência recebida nos atendimentos. Os profissionais foram considerados preparados por $75 \%$ dos cuidadores. A utilização de estabilização física foi necessária em $50 \%$ dos casos. A maior parte dos atendimentos foram realizados por dentistas não especializados em Pacientes com Necessidades Especiais (62,5\%) e os consultórios não eram adaptados (60,0\%). Considerações finais: A utilização dos serviços odontológicos pelo paciente e do cuidador devem ser considerados propósitos a serem alcançados por qualquer serviço de saúde, pois representam um monitoramento das ações ofertadas aos olhos daqueles que os procuram.
\end{abstract}

Palavras-chave: Assistência odontológica; Síndrome de Down; Odontopediatria.

\begin{abstract}
Objective: To evaluate the use of dental services by pediatric patients with Down Syndrome according to caregivers. Methodology: A cross-sectional study was carried out at the Fundação Centro Integrada de Support to the Disabled (FUNAD), in João Pessoa-PB. The sample consisted of 49 caregivers of patients with Down syndrome, between 0 and 15 years old. Socioeconomic and satisfaction data about the service offered were collected using a form designed in
\end{abstract}


accordance with the research objectives. Results: It was observed that $83.7 \%$ of the patients have already been to the dentist, more frequently in the Basic Health Unit (47.5\%). 97.5\% of the caregivers were satisfied with the assistance received in the visits. The professionals were considered prepared by $75 \%$ of the caregivers. The use of physical stabilization was necessary in $50 \%$ of the cases. Most consultations were performed by dentists who were not specialized in Patients with Special Needs (62.5\%) and the offices were not adapted (60\%). Final considerations: The use of dental services by the patient and the caregiver must be considered purposes to be achieved by any health service, as they represent a monitoring of the actions offered in the eyes of those who seek them.

Keywords: Dental care; Down syndrome; Pediatric dentistry.

\section{Resumen}

Objetivo: Evaluar el uso de los servicios dentales por parte de los pacientes pediátricos con Síndrome de Down según los cuidadores. Metodología: Se realizó un estudio transversal en la Fundación Centro Integrada de Apoyo a Discapacitados (FUNAD), en João Pessoa-PB. La muestra estuvo formada por 49 cuidadores de pacientes con Síndrome de Down, entre 0 y 15 años. Los datos socioeconómicos y de satisfacción sobre el servicio ofrecido se recogieron mediante un formulario elaborado de acuerdo con los objetivos de la investigación. Resultados: Se observó que el $83,7 \%$ de los pacientes ya han acudido al odontólogo, con mayor frecuencia en la Unidad Básica de Salud (47,5\%). El 97,5\% de los cuidadores se mostraron satisfechos con la asistencia recibida en las visitas. Los profesionales fueron considerados preparados por el $75 \%$ de los cuidadores. El uso de estabilización física fue necesario en el $50 \%$ de los casos. La mayoría de las consultas fueron realizadas por odontólogos no especializados en Pacientes con Necesidades Especiales $(62,5 \%)$ y los consultorios no fueron adaptados (60\%). Consideraciones finales: La utilización de los servicios odontológicos por parte del paciente y del cuidador debe ser considerada fines a alcanzar por cualquier servicio de salud, ya que representan un seguimiento de las acciones ofrecidas a los ojos de quienes las buscan.

Palabras clave: Atención odontológica; Síndrome de Down; Odontología pediátrica.

\section{Introdução}

A Síndrome de Down (SD) é uma doença genética que ocorre quando há uma alteração de forma atípica na separação dos cromossomos 21, podendo atingir em torno de 1/700 dos nascidos. Dessa forma, o indivíduo afetado apresenta deficiência intelectual, motora e na linguagem, além de doença ou comorbidades associadas (Aguiar, Barbieri, \& Castro, 2003; Bermudez, Medeiros, Bermudez, Novadzki, \& Magdalena, 2015; Lowy, 2019).

A resolução 22/2001, Artigo 31, do Conselho Federal de Odontologia (CFO), afirma que a especialidade de Pacientes com Necessidades Especiais (PNE) tem o objetivo de diagnosticar, prevenir, tratar e controlar os problemas de saúde desses indivíduos. De acordo a Portaria n ${ }^{\circ}$ 599, de 23 de março de 2006, o atendimento de média complexidade aos pacientes com necessidades especiais pode ser realizado nos Centros de Especialidades Odontológicas (CEO), os quais são definidos como estabelecimentos especializados em saúde bucal, com ênfase no diagnóstico de câncer bucal, doenças periodontais, cirurgia oral menor dos tecidos moles e duros, endodontia e atendimentos a pessoas com necessidades especiais. No entanto, o atendimento a esse paciente também pode acontecer na baixa e na alta complexidade, de acordo com as necessidades do mesmo (Brasil, 2004; Pini, Frohlich, \& Rigo, 2016).

Para que ocorra um melhor atendimento e acolhimento dos pacientes com a SD, é necessário o conhecimento das características clínicas e das alterações sistêmicas que eles possuem (Caldas Júnior \& Machiavelli, 2013). No que se refere aos aspectos bucais de interesse odontológico, alguns autores afirmam que indivíduos com estas condições, em sua maioria, são respiradores bucais, o que pode estar relacionado à atresia do palato duro e, em alguns casos, com fendas labial e palatina (Teitelbaum \& Sabbagh-Haddad, 2007). É muito comum observar a ocorrência de más oclusões Classe III de Angle, o que pode ocasionar as mordidas cruzadas anterior e posterior e bruxismo (Mugayar, 2000; Andersson, Axelsson, \& Katsaris, 2016).

Além disso, a cárie dentária e principalmente a doença periodontal são agravos bucais frequentes em pacientes pediátricos com SD, isso torna a condição de saúde bucal desses pacientes preocupante, visto que estes agravos determinam impacto negativo na qualidade de vida dos pacientes e familiares (Habashneh, Al-Jundi, Khader, \& Nofel, 2012; Carrada, Scalioni, Abreu, Ribeiro, \& Paiva, 2020). 
Pacientes com SD requerem atendimento odontológico precoce, afim de prevenir e limitar o aumento da gravidade de possíveis agravos bucais (Peinado, Martínez, Pérez, \& García, 2018). Dessa forma, é válido ressaltar que se trata de seres humanos e que, cada um, de forma exclusiva, tem suas limitações estruturais e emocionais, deste modo, os cirurgiões-dentistas devem sempre respeitar suas limitações (Fonseca, Azzalis, Fonseca, \& Botazzo, 2010; Descamps, Fernandez, Van Cleynenbreugel, Van Hoecke, \& Marcas, 2019).

Esse estudo partiu da necessidade de avaliar a percepção do atendimento odontológico ofertado aos pacientes com SD, ressaltada pela escassez de publicações voltadas para esta área. Para tanto, objetivou-se neste trabalho avaliar a percepção dos cuidadores acerca do acesso e satisfação com o serviço odontológico ofertado aos pacientes pediátricos com SD.

\section{Metodologia}

Trata-se de uma pesquisa quantitativa, que de acordo com Rodrigues (2007), traduz em números as opiniões e informações para serem classificadas e analisadas. Apresenta caráter observacional, pois não houve intervenção na população estudada, e o estudo teve desenho transversal, realizado no período do mês de março de 2018.

A pesquisa foi desenvolvida nas dependências da FUNAD, Órgão do Governo do Estado da Paraíba, referência no Serviço de Habilitação e Reabilitação física, intelectual, visual e auditiva, em todo o Estado, que fornece atendimento multidisciplinar aos pacientes.

Foi realizado um censo com 56 cuidadores de pacientes pediátricos com SD, destes, dois cuidadores se recusaram a participar da pesquisa e cinco não estavam comparecendo a instituição no dia de atendimento do paciente após três tentativas sucessivas, dessa forma, a taxa de resposta foi de $87,5 \%$.

Utilizou-se como critérios de elegibilidade os cuidadores de pacientes pediátricos com SD, na faixa etária de 0 a 15 anos, com matrícula ativa na FUNAD.

A coleta foi realizada em uma sala de atendimento da FUNAD, reservada para a coleta de dados, por meio da aplicação de um formulário que possibilitou a utilização dos serviços odontológicos por pacientes pediátricos com SD. O instrumento de coleta de dados foi constituído por vinte e nove (29) questões objetivas, desenvolvido pela equipe da pesquisa.

Os dados quantitativos foram analisados descritivamente. A análise inferencial também foi realizada por meio do teste qui-quadrado para comparação da satisfação com o atendimento odontológico, de acordo com o local de atendimento $(\alpha<0,05)$, por meio do software Statistical Package for the Social Sciences (SPSS), versão 20.

O estudo seguiu a Resolução no 466/12-CNS/MS, tendo início após a aprovação do Comitê de Ética em Pesquisa do UNIPÊ (CAAE - 80009817.6.0000.5176).

\section{Resultados}

Participaram do estudo 49 cuidadores de pacientes pediátricos com SD, logo, a taxa de resposta foi de 87,5\%. Sete não participaram, pois dois se recusaram e cinco não estavam frequentando a instituição.

Os dados demográficos e socioeconômicos foram descritos na Tabela 1. Observou-se que a maioria dos cuidadores eram do sexo feminino (89,8\%), com idade entre 25 e 56 anos. A escolaridade de ensino fundamental incompleto $(40,8 \%)$ apresentou maior percentual, em relação às demais.

Observa-se também que $71,4 \%$ da amostra possuía uma renda familiar de 1 a 2 salários mínimos. Os pacientes pediátricos, em 57,1\%, eram residentes de outras cidades do interior do estado da Paraíba, sendo a casa coberta pela Estratégia da Saúde em Família, em cerca de 93,9\% da amostra. 
Tabela 1 - Perfil demográfico e socioeconômico dos cuidadores. João Pessoa, 2018.

\begin{tabular}{|c|c|c|}
\hline \multirow[t]{2}{*}{ Variáveis } & \multicolumn{2}{|c|}{ Frequência relativa } \\
\hline & $\mathbf{N}$ & $(\%)$ \\
\hline \multicolumn{3}{|l|}{ Sexo do cuidador $(n=49)$} \\
\hline Feminino & 44 & 89,8 \\
\hline Masculino & 05 & 10,2 \\
\hline \multicolumn{3}{|l|}{ Idade do cuidador $(n=49)$} \\
\hline 25 a 40 & 26 & 53,1 \\
\hline 41 a 56 & 23 & 46,9 \\
\hline \multicolumn{3}{|l|}{ Grau de parentesco do cuidador $(n=49)$} \\
\hline Mãe & 38 & 77,5 \\
\hline Pai & 06 & 12,2 \\
\hline Avó/Avô & 05 & 10,3 \\
\hline \multicolumn{3}{|l|}{ Grau de escolaridade do cuidador $(n=49)$} \\
\hline Não Alfabetizado-Ensino Fundamental Incompleto & 20 & 40,8 \\
\hline Ensino Fundamental Completo-Ensino Médio Incompleto & 09 & 18,4 \\
\hline Ensino Médio Completo & 14 & 28,6 \\
\hline Ensino Superior Incompleto-Ensino Superior Completo & 06 & 12,2 \\
\hline \multicolumn{3}{|l|}{ Renda familiar $(n=49)$} \\
\hline Menos de 1 salário mínimo & 05 & 10,2 \\
\hline De 1 a 2 salários mínimos & 35 & 71,4 \\
\hline De 2 a 3 salários mínimos & 08 & 16,3 \\
\hline De 3 a 5 salários mínimos & 01 & 2,1 \\
\hline \multicolumn{3}{|l|}{ Cidade $(n=49)$} \\
\hline João Pessoa & 21 & 42,9 \\
\hline Outras cidades & 28 & 57,1 \\
\hline \multicolumn{3}{|l|}{ Área acobertada pelo PSF $(n=49)$} \\
\hline $\operatorname{Sim}$ & 46 & 93,9 \\
\hline Não & 03 & 6,10 \\
\hline
\end{tabular}

Fonte: Autores (2018).

Os dados da Tabela 2 descreveram a utilização do serviço odontológico por parte das crianças e adolescentes com SD, 83,7\% dos pacientes já tinham ido ao dentista. A faixa etária em que os pacientes foram pela primeira vez ao dentista, mais frequente, foi de 6 a 11 anos (83,7\%). A última consulta com o dentista foi há menos de 1 ano (75\%). Dentre os que foram ao dentista, 70\% tiveram sua consulta odontológica realizada no setor público. A maior parte dos atendimentos odontológicos foram realizados na Unidade Básica de Saúde (UBS) (47,5\%), seguido do CEO (20,0\%). Durante a procura de consulta odontológica, $75,5 \%$ não tiveram dificuldade para obter a consulta odontológica. 
Tabela 2 - Descrição da utilização ao serviço odontológico por pacientes pediátricos com SD. João Pessoa, 2018.

\begin{tabular}{lcr}
\hline Variáveis & \multicolumn{2}{c}{ Frequência rel } \\
& $\mathbf{N}$ & $(\%)$ \\
\hline Ida ao dentista $(\mathbf{n}=\mathbf{4 9})$ & & 83,7 \\
Sim & 40 & 16,3 \\
Não & 09 &
\end{tabular}

\section{Motivo da não ida ao dentista (n=9)}

Cuidador achou que não precisava

Não achou atendimento

Criança com problema sistêmico

Local do último atendimento $(n=40)$

Unidade de Saúde Básica

Centro de Especialidades Odontológicas (CEO)

Clínica Escola

10,0

Consultório particular

Dificuldade para encontrar dentista $(n=49)$

Sim

Não

Tipo de dificuldade $(\mathbf{n}=11)$

Não conseguiu atendimento no CEO

Falta de dentista constantemente

Recusa do dentista do PSF

Idade da primeira consulta $(n=40)$

0 a 2 anos

3 a 5 anos

6 a 11 anos

55,0

Acima de 12 anos

Último atendimento $(n=40)$

1 a 11 meses

12 ou mais meses

Motivo da última consulta $(n=40)$

Avaliação

Cárie dentária

Exodontia

Inflamação gengival

Dentes rangidos

Dentes decíduos não esfoliados

Recusa do atendimento $(n=40)$

Sim

Não

Motivo da recusa $(n=3)$

Falta de material 
Os dados da Tabela 3 descreveram a satisfação dos cuidadores de pacientes com SD a respeito dos serviços odontológicos ofertados aos mesmos. $60 \%$ dos consultórios não eram adaptados para receber pessoas com deficiência, entretanto, 77,5\% dos cirurgiões-dentistas se mostraram ter domínio sobre esta área. Em 50\% dos casos, não foi necessário o uso de estabilização física, durante a consulta e a maioria teve o seu tratamento concluído com sucesso (75\%). Aqueles que não concluíram, foi porque o tratamento ainda está em andamento (90\%) ou foi porque o próprio paciente não permitiu a conclusão, por causa da sua não colaboração (10\%).

No que diz respeito a opinião e satisfação dos cuidadores com a consulta realizada nos pacientes pediátricos, $97,5 \%$ afirmaram terem sido bem atendidos, terem recebido a assistência necessária e terem ficado satisfeitos com o atendimento. Foi perguntado como os mesmos avaliavam esta consulta odontológica e, 42,5\% responderam "muito bom", sendo o cirurgião dentista avaliado com uma nota de 8 a 10 em $92,5 \%$ dos casos.

Tabela 3 - Descrição da satisfação com os serviços odontológicos ofertados aos pacientes pediátricos com SD. João Pessoa, 2018.

\begin{tabular}{lcc}
\hline Variáveis & \multicolumn{2}{c}{$\begin{array}{c}\text { Frequência relativa } \\
(\%)\end{array}$} \\
\hline O CD era especializado em PNE na última consulta? (n=40) & N & 37,5 \\
Sim & 15 & 62,5 \\
Não & 25 & 40,0 \\
O consultório era adaptado na última consulta? (n=40) & 16 & 60,0 \\
Sim & 24 & \\
Não & & 77,5 \\
O CD tinha domínio sobre a SD na última consulta? (n=40) & 31 & 22,5 \\
Sim & 09 & \\
Não & & 50 \\
Usou de estabilização (n=40) & 20 & 47,5 \\
Não foi necessário & 19 & 2,5 \\
Estabilização física ativa e passiva & 01 & \\
Anestesia geral & & 75,0 \\
O tratamento odontológico foi concluído (n=40) & 30 & 25,0 \\
Sim & 10 & \\
Não & & 90,0 \\
Motivo da não conclusão (n=10) & 09 & 10,0 \\
Tratamento em andamento & 01 & \\
Paciente não deixou & & \\
Ficou satisfeito com o tratamento? (n=40) & 39 & 97,5 \\
Sim & 01 & 2,5 \\
Não & & \\
Avaliação do atendimento odontológico (n=40) & 17 & 42,5 \\
Ótimo & 22 & 55,0 \\
Regular & 01 & 2,5 \\
Ruim &
\end{tabular}

Fonte: Autores (2018).

A frequência da satisfação não apresentou diferença significativa de acordo com o setor, conforme descrito na Tabela 4. 
Tabela 4 - Satisfação com o atendimento odontológico de acordo com o local de atendimento. João Pessoa, 2018.

\begin{tabular}{llll}
\hline Local do atendimento & \multicolumn{2}{c}{ Satisfação com o tratamento } & \\
& Sim n (\%) & Não n (\%) & Valor de p \\
\hline Consultório Particular & $8(100)$ & $0(0)$ & 0,769 \\
Unidade de Saúde Básica & $18(94,7)$ & $1(5,3)$ & \\
CEO & $9(100)$ & $0(0)$ & \\
Clínicas escolas & $4(100)$ & $0(0)$ & \\
\hline
\end{tabular}

Teste qui-quadrado. Fonte: Autores (2018).

\section{Discussão}

O foco principal do presente estudo foi avaliar o acesso e satisfação dos cuidadores com os serviços odontológicos, sejam eles públicos ou privados, ofertados aos pacientes com SD. Considerando-se a necessidade de minimizar a perda de informações ou amostras, durante a coleta de dados, utilizou-se um formulário como instrumento de pesquisa, preenchido por meio de entrevista com o cuidador.

A maior parte dos cuidadores eram do sexo feminino, apesar dos pacientes terem sido com maior frequência do sexo masculino, o que não diferiu de outros estudos, sendo a mãe o cuidador mais frequente (Chaves, Cruz, Barros, \& Figueiredo, 2011; Saliba, Nayme, Moimaz, Cecilio, \& Garbin, 2013; Ronca, Rocha, Pozzi, Cymrot, \& Blascovi-Assis, 2017), sendo mais frequente na sociedade, as mulheres assumirem o cuidar do lar (Pinto, Matos, \& Loyola Filho, 2012).

Segundo o presente estudo, a maior proporção de usuários tinha uma renda familiar baixa, de aproximadamente 1 a 2 salários mínimos. Uma pesquisa anterior, realizada em Olinda-PE mostrou resultado semelhante, em um estudo realizado nos CEOs (Oliveira, 2008).

Com relação a escolaridade das crianças, o ensino fundamental incompleto $(85,7 \%)$ foi o mais frequente e em concordância com este dado, o estudo de Oliveira, Fragoso, Silva, Brito e Cardoso (2019), realizado com cuidadores de crianças e adolescentes com deficiência auditiva mostrou que a maioria das crianças e adolescentes (50,0\%) também apresentavam ensino fundamental incompleto. A diferença de porcentagem com o presente estudo pode estar associada ao tipo de deficiência e dificuldade de acesso.

Neste estudo, a maioria dos cuidadores relataram que os pacientes já haviam ido ao dentista em outro momento. Um estudo anterior também mostrou que a maioria das crianças com deficiência motora já tinham tido acesso ao atendimento odontológico (Cardoso, Brito, Alves, \& Padilha, 2011). Em outro estudo, 85\% de PNE, já tinham tido acesso ao atendimento odontológico (Al Agili, Roseman, Pass, Thornton, \& Chavers, 2004).

Esta pesquisa também mostrou que grande parte das crianças e adolescentes utilizaram o serviço há menos de um ano, com tratamentos concluídos ou em andamento. $\mathrm{O}$ aumento de contratação de cirurgião-dentista na rede pública ampliou o acesso ao serviço odontológico, sendo o Nordeste, a região com maior número de contratação, ou seja, muitos cirurgiõesdentistas estão atuando na rede pública dando assistência na atenção básica (Antunes, \& Narvai, 2010).

Neste estudo, poucos cuidadores relataram sentir dificuldade para conseguir o atendimento odontológico. Um estudo anterior, realizado na cidade de João Pessoa, mostrou que 65\% dos cuidadores relataram encontrar dificuldades em conseguir atendimento odontológico para PNE (Cardoso et al., 2011). No entanto, no Brasil, em 2011 foi lançado o plano nacional dos direitos a pessoa com deficiência, Plano Viver sem Limites que instituiu várias ações na área de Odontologia com o intuito de melhorar o acesso ao serviço odontológico público. Dentre elas, a capacitação de 6 mil equipes das Unidades Básicas de Saúde (UBS), qualificação de $420 \mathrm{CEOs}$, com carga horária obrigatória de 40 horas com dentista especializado em PNE e criação de 
27 centros cirúrgicos (Brasil, 2013), o que pode justificar esse aumento do acesso na rede pública. Além disso, aumentou a inserção da disciplina de Pacientes com Necessidades Especiais nos cursos de graduação em Odontologia, o que pode ter favorecido o aumento da utilização dos serviços odontológicos pelos PNEs.

Embora a menor parte dos cuidadores tenham sentido dificuldade na utilização ao serviço odontológico, estes relataram que a principal dificuldade foi conseguir agendar o atendimento no CEO. Um estudo realizado em Bauru aponta que essa dificuldade pode ser justificada pela alta demanda de PNEs atendidos nesses serviços, além da dificuldade de contratação de profissionais especialistas ou interessados nessa área (Nacamura, Yamashita, Busch, \& Nader Marta, 2015). Portanto, observou uma melhora na logística da oferta do serviço odontológico, porém alguns pacientes ainda encontram dificuldade, de acordo com as características do local, do profissional e do paciente.

Esse estudo mostrou que a faixa etária mais frequente em que as crianças e adolescente realizaram a primeira consulta odontológica foi de 6 a 11 anos, o que mostra que esses pacientes não realizaram sua primeira consulta odontológica até a idade de 6-12 meses recomendada pela American Academy of Pediatric Dentistry (1989). A literatura mostra que existe a necessidade de iniciar a abordagem odontológica precocemente, fazendo com que se priorizem os procedimentos preventivos (Miotoo, Bortolo, \& Araújo, 2003), no entanto, isso não aconteceu com a população estudada.

O problema odontológico mais relatado pelos cuidadores que motivou a consulta foi à cárie dentária. Segundo estudo anterior, as pessoas com deficiência sentem dificuldade para manter uma boa saúde bucal ou ter acesso aos serviços odontológicos (Castro, Marchesoti, Oliveira, \& Novaes, 2010). Os agravos mais frequentes em pacientes com SD são cárie dentária, devido a higienização bucal deficiente, e doença periodontal, esta se apresenta com prevalência aumentada, em virtude da possível presença de fatores locais e sistêmicos como higiene bucal reduzida, presença de cálculo, macroglossia, morfologia dental, microbiota subgengival periodontopatogênica, alteração no tecido gengival, saliva e função imunológica (Nacamura et al., 2015; Mubayrik, 2016). No entanto, ambos agravos já citados podem ser prevenidos com a realização de um cuidado bucal satisfatório e atencioso.

De acordo com os cuidadores deste estudo, alguns cirurgiões-dentistas recusaram-se a realizar o atendimento no paciente com SD, e os principais motivos eram a insegurança do profissional e falta de material. Um estudo anterior, relatou que os cirurgiões-dentistas atendiam os PNE's, mas realizavam procedimentos menos invasivos, como: exame clínico, profilaxias e aplicação de flúor (Lawrence, Sousa, Gonçalves, Saintrain, \& Vieira, 2014). Dessa forma, uma parte da população pode continuar com necessidades odontológicas não atendidas, apesar de ter procurado a consulta odontológica.

A maioria dos cuidadores estavam satisfeitos com o atendimento que os pacientes pediátricos com SD receberam, seja ele na UBSs, CEOs, clínicas-escolas ou nos consultórios particulares. Esse resultado positivo foi em concordância com uma pesquisa realizada em uma clínica-escola do estado do Pernambuco, onde obtiveram um resultado de $95,4 \%$ de satisfação (Oliveira, Martins, Santana, Bezerra, \& Dourado, 2012).

De acordo com um estudo anterior, com relação a percepções de cirurgiões-dentistas envolvidos no atendimento de PNEs de serviços públicos municipais, os profissionais relataram não conseguir suprir com a demanda e a necessidade dos pacientes, os autores ainda ressaltaram que esse fato pode estar ligado a um conjunto de sentimentos (medo, ansiedade e expectativas) inerentes a qualquer profissional que atende um PNE (Fonseca, Azzalis, Fonseca, \& Botazzo, 2010).

Durante a entrevista, foi questionado como os usuários avaliavam o atendimento odontológico realizado nos pacientes. O maior percentual de resposta foi "muito bom", resultado semelhante a estudos realizados nos estados de Minas Gerais (Minas, 2002) e do Rio Grande do Sul (Grando, \& Hashizume, 2019), como também, em um estudo realizado com os usuários dos centros de saúde comunitários Zapotitla e San José, no México (Flores, Cámara, Estrella, \& Velásquez, 2000). A literatura relata que essa satisfação positiva alcançada não é mérito, exclusivamente, do trabalho técnico do cirurgião-dentista, 
pois o atendimento odontológico também é julgado por outros aspectos, como a confiança passada aos responsáveis dos pacientes e o preparo das assistentes (Grando, \& Hashizume, 2019).

Além disso, um estudo anterior relatou que a grande satisfação dos usuários com o atendimento odontológico, se deve, principalmente, a um perfil diferente do profissional, onde o mesmo geralmente explica o tratamento a ser realizado e desenvolve uma relação empática e participativa com eles (Lima, Cabral, \& Vasconcelos, 2010).

É importante ressaltar que a boa percepção com os serviços odontológicos pode ter ocorrido devido as condições limitadas dos cuidadores e pacientes em frequentar outros serviços, ficando intimidados a responder positivamente a respeito do mesmo, com receio de que isso viesse a prejudicá-lo na sua acessibilidade, por parte dos profissionais que, aos olhos deles, poderiam ter autonomia para isso, como ocorreu da mesma forma com um estudo realizado por usuários adultos para avaliar o nível de satisfação oferecida pela clínica integrada de uma Faculdade de Odontologia no Piauí (Pompeu, Carvalho, Pereira, Cruz Neto, Prado, \& Silva, 2012).

Durante a pesquisa, foi verificado que, na metade dos casos, não foi necessário o uso de nenhum meio de estabilização física, durante o atendimento odontológico da criança. Isso pode ser justificado pelo fato de que muitos pacientes com deficiência não compreendem a necessidade do atendimento odontológico (Barrêto, Oliveira, Paiva, \& Pordeus, 2004).

Este estudo apresentou um desenho transversal, sendo necessária a realização de estudos longitudinais que possam acompanhar o seguimento da satisfação com o serviço odontológico, como também, os dados coletados podem apresentar o viés de memória, já que o atendimento odontológico pode ter acontecido há algum tempo, o que favorece uma falha na memória do cuidador. Além disso, trata-se de um estudo local, o que sugere que novas pesquisas sejam realizadas em outras regiões do Brasil.

Assim, observou-se o que o serviço odontológico ofertado aos pacientes com SD, mesmo o cirurgião-dentista não sendo especializado na área de PNE e com todas as restrições de um serviço básico, foi bem avaliado. A formação de um profissional com um olhar voltado não apenas para a cavidade bucal, e sim, para todo o indivíduo pode ser consequência de medidas tomadas durante a graduação de Odontologia ao implementar a disciplina de PNE na matriz curricular, assegurando que o profissional saia mais preparado para atender qualquer tipo de público no seu âmbito profissional e/ou pelas esferas de governo que tentam atender a legislação que garante a todo o cidadão o direito à saúde.

Os resultados deste estudo podem orientar gestores de saúde e profissionais no planejamento de estratégias de cuidado odontológico que proporcionem melhor saúde bucal e, consequentemente, melhorar o acesso, satisfação, bem como a qualidade de vida dos pacientes com SD e cuidadores.

\section{Considerações Finais}

Um grande percentual dos cuidadores de pacientes pediátricos com SD estavam satisfeitos com o serviço odontológico ofertado, sendo o serviço público mais frequentado. Os cuidadores apresentaram condições socioeconômicas baixas e ensino fundamental incompleto. A maioria se mostrou bastante satisfeito com a conduta do CD, mesmo trabalhando em um local que não oferecia uma infraestrutura adequada para um PNE, porém, as dificuldades relatadas por aqueles cuidadores que não conseguiram acesso ao serviço odontológico foram: não conseguir agendar o atendimento, falta de CD no dia do atendimento agendado e a recusa de alguns destes profissionais para atender esse público de pacientes.

A utilização do paciente e do cuidador aos serviços odontológicos deve ser considerado um propósito a ser alcançado por qualquer serviço de saúde, pois representa um monitoramento das ações ofertadas aos olhos daqueles que os procuram. 


\section{Referências}

Aguiar, S. M. H. C. A., Barbieri, C. M., \& Castro, A. M. (2003). Incidência da síndrome de Down associada à idade materna mais avançada. J Bras OdontoPsicol Odontol Pacientes Espec., 1(2), 166-168.

Al Agili, D. E., Roseman, J., Pass, M. A., Thornton, J. B., \& Chavers, L. S. (2004). Access to dental care in Alabama for children with special needs: parents' perspectives. J Am Dent Assoc., 135(4), 490-495.

Al Habashneh, R., Al-Jundi, S., Khader, Y., \& Nofel, N. (2012). Oral health status and reasons for not attending dental care among 12- to 16-year-old children with Down syndrome in special needs centres in Jordan. Int J Dent Hyg., 10(4), 259-64.

American Academy of Pediatric Dentistry (1989). Reference Manual 1991-1992: Infant oral health care.p 30.

Andersson, E. M., Axelsson, S., \& Katsaris, K. P. (2016). Malocclusion and the need for orthodontic treatment in 8-year-old children with Down syndrome: a cross-sectional population-based study. Spec Care Dentist. 36(4), 194-200.

Antunes, J. L. F., \& Narvai, P. C. (2010). Políticas de saúde bucal no Brasil e seu impacto sobre as desigualdades em saúde. Rev. Saúde Pública [online], 44(2), 360-365.

Barrêto, A. P. R., Oliveira, C. S., Paiva, S. M., \& Pordeus, I. A. (2004). Qualidade de vida infantil: influência dos hábitos de higiene bucal e do acesso aos serviços odontológicos. Rev Ibero-Amer Odontop Odontol Bebê, 7(39), 453-460.

Bermudez, B. E. B. V., Medeiros, S. L., Bermudez, M. B., Novadzki, I. M., \& Magdalena, N. I. R. (2015). Down syndrome: prevalence and distribution of congenital heart disease in brazil. São Paulo Medical Journal, 133(6), 521-524.

Caldas Júnior, A. F., \& Machiavelli, J. L (2013). Atenção e Cuidado da Saúde Bucal da pessoa com Deficiência: protocolos, diretrizes e condutas para cirurgiões-dentistas: Ed. Universitária.

Castro, A. M., Marchesoti, M. G. N., Oliveira, F. S., \& Novaes, M. S. P. (2010). Avaliação do tratamento odontológico de pacientes com necessidades especiais sob anestesia geral. Rev Odontol UNESP, 39(3), 137-142.

Cardoso, A. M. R., Brito, D. B. A., Alves, V. F., \& Padilha, W. W. N. (2011). O Acesso ao Cuidado em Saúde Bucal para Crianças com Deficiência Motora: Perspectivas dos Cuidadores. Pesq Bras Odontoped Clin Integr., 11(4), 593-599.

Carrada, C. F., Scalioni, F. A. R., Abreu, L. G., Ribeiro, R. A., \& Paiva, S. M. (2020). Impact of oral conditions of children/adolescents with Down syndrome on their families' quality of life. Spec Care Dentist., 40(2), 175-183.

Chaves, S. C. L., Cruz, D. N., Barros, S. G., \& Figueiredo, A. L. (2011). Avaliação da oferta e utilização de especialidades odontológicas em serviços públicos de atenção secundária na Bahia, Brasil. Cad Saúde Pública, 27(1), 143-154.

Descamps, I., Fernandez, C., Van Cleynenbreugel, D., Van Hoecke, Y., \& Marcas, L. (2019). Dental care in children with Down syndrome: A questionnaire for Belgian dentists. Med Oral Patol Oral Cir Bucal, 24(3), 385-391.

Flores, N. L., Cámara, V. L., Estrella, S. L. M., \& Velásquez, E. C. (2000). Satisfacción de usuarios de dos servicios odontológicos en Tláhuac, Distrito Federal. Revista ADM, 57(5):45-49.

Fonseca, A. L. A., Azzalis, L. A., Fonseca, F. L. A., \& Botazzo, C. (2010). Análise qualitativa das percepções de cirurgiões-dentistas envolvidos nos atendimentos de pacientes com necessidades especiais de serviços públicos municipais. Rev Bras Crescimento e Desenvolvimento Hum., 20 (2), $208-216$.

Grando, D., \& Hashizume, L. N. (2019). Avaliação do acesso e satisfação aos serviços odontológicos prestados a indivíduos com síndrome de Down na visão de seus pais ou cuidadores. Rev. Fac. Odontol. Porto Alegre, 60(2), 11-19.

Lawrence, H., Sousa, L. P., Gonçalves, F. L., Saintrain, M. V. L., \& Vieira, A. P. G. F. (2014). Acesso à saúde bucal pública pelo paciente especial: a ótica do cirurgião-dentista. Revista Brasileira em Promoção da Saúde, 27(2), 190-197.

Lima, A. C. S., Cabral, E. D., \& Vasconcelos, M. M. V. B. (2010). Satisfação dos usuários assistidos nos Centros de Especialidades Odontológicas do Município de Recife, Pernambuco, Brasil. Cad de Saúde Pública, 26(5), 991-1002.

Lowy, I. (2019). How diseases became "genetic". Cien Saude Colet., 24(10), 3607-3617.

Ministério da Saúde. Secretaria de Políticas de Saúde. Agenda de Compromisso para saúde integral da criança e redução da mortalidade infantil. Brasília (DF).

Ministério da Saúde. Secretaria Nacional de Promoção dos direitos da pessoa com Deficiência. Plano viver sem limites - Plano Nacional dos direitos da pessoa com Deficiência. Brasília [DF].

Minas, R. P. (2002). O Paciente no contexto do ensino [dissertação]. [Belo Horizonte]: Universidade Federal de Minas Gerais.

Miotto, M. H. M. B., Bortolo, D. P., \& Araújo, M. P. G. (2015). Manejo odontológico de paciente deficiente auditivo. Relato de caso. Revista Brasileira De Pesquisa Em Saúde/Brazilian Journal of Health Research, 5(1): 24-31.

Mubayrik, A. B. (2016). The Dental Needs and Treatment of Patients with Down Syndrome. Dent Clin North Am., 60(3), 613-26.

Mugayar, L. R. F. (2000). Pacientes portadores de necessidades especiais: manual de odontologia e saúde oral: Pancast.

Nacamura, C. A., Yamashita, J. C., Busch, R. M. C., \& Nader Marta, S. (2015). Síndrome de Down: inclusão no atendimento odontológico municipal. Revista da Faculdade de Odontologia de Lins, 25(1), 27-35. 
Research, Society and Development, v. 10, n. 4, e9010413767, 2021

(CC BY 4.0) | ISSN 2525-3409 | DOI: http://dx.doi.org/10.33448/rsd-v10i4.13767

Oliveira, J. J. M., Fragoso, K. T., Silva, M. R. L., Brito, C. S. M., \& Cardoso, A. M. R. (2019). Utilização do serviço odontológico por crianças e adolescentes com deficiência auditiva e sua comunicação com o cirurgião dentista. Revista de Iniciação Científica em Odontologia, 2(17), 25-35.

Oliveira, O. R., Martins, E. P., Santana, J. L. B., Bezerra, S. R. S., \& Dourado, A. T. (2012). Avaliação dos usuários sobre a qualidade do atendimento odontológico prestado por alunos de Odontologia. RFO UPF [online], 17(3), 319-325.

Oliveira, R. S. (2008). Acesso aos serviços de saúde bucal e avaliação da satisfação do usuário em Olinda - PE [monografia]. [Campina Grande]: Faculdade de Ciências Sociais e Aplicadas.

Peinado, N. R., Martínez, M. R. M., Pérez, M. D., \& García, M. J. N. (2018). A study of the dental treatment needs of special patients: cerebral paralysis and Down syndrome. Eur J Paediatr Dent., 19(3), 233-238.

Pini, D. M., Frohlich, P. C., \& Rigo, L. (2016). Oral health evaluation in special needs individuals. Einstein (São Paulo), 14(4), 501-507.

Pinto, R. S., Matos, D. L., \& Loyola Filho, A. I. (2012). Características associadas ao uso de serviços odontológicos públicos pela população adulta brasileira. Cienc Saúde Coletiva, 17(2), 531-544.

Pompeu, J. G. F., Carvalho, I. L. M., Pereira, J. Á., Cruz Neto, R. G., Prado, V. L. G., \& Silva, C. H. V. S. (2012). Avaliação do nível de satisfação dos usuários atendidos na clínica integrada do curso de Odontologia da Faculdade Novafapi em Teresina (PI). Odontol Clín-Cient., 11(1), 31-36.

Rodrigues, W. C. (2007). Metodologia Científica. Rev. Guanabara Koogan, Paracambi, jun./jul.

Ronca, R. P., Rocha, M. M., Pozzi, D. C., Cymrot, R., \& Blascovi-Assis, S. M. (2019). Síndrome de Down: irmãos fazem diferença na qualidade de vida dos pais?. Psicologia em Estudo, 24, 2-17.

Saliba, N. A., Nayme, J. G. R., Moimaz, S. A. S., Cecilio, L. P. P., \& Garbin, C. A. S. (2013). Organização da demanda de um Centro de Especialidades Odontológicas. Rev Odontol UNESP, 42(5), 317-323.

Teitelbaum, A. P., \& Sabbagh-Haddad, A. (2007). Alteração sistêmica na Síndrome de Down e cuidados no atendimento odontológico. Rev Assoc Paul Dent., 61(3), 237-42. 